\title{
Self-excited air flow passage changing device for periodic pressurization of soft robot
}

\author{
Toshio Takayama* ${ }^{*}$ and Yusuke Sumi
}

\begin{abstract}
Recently pneumatic-driven soft robots have been widely developed. Usually, the operating principle of this robot is the inflation and deflation of elastic inflatable chambers by air pressure. Some soft robots need rapid and periodic inflation and deflation of their air chambers to generate continuous motion such as progress motion or rotational motion. However, if the soft robot needs to operate far from the air pressure source, long air tubes are required to supply air pressure to its air chambers. As a result, there is a large delay in supplying air pressure to the air chamber, and the motion of the robot slows down. In this paper, we propose a compact device that changes its airflow passages by self-excited motion generated by a supply of continuous airflow. The diameter and the length of the device are 20 and $50 \mathrm{~mm}$, respectively, and can be driven in a small pipe. Our proposed in-pipe mobile robot is connected to the device and can move in a small pipe by dragging the device into it. To apply the device widely to other soft robots, we also discuss a method of adjusting the output pressure and motion frequency.
\end{abstract}

Keywords: Self-excited valve, In-pipe robot, Soft robot

\section{Introduction}

The advantages of a pneumatically actuated device are not only its high power-to-weight ratio but also the absence of any mechanical elements that use electromagnetic force at the end effector of the device. Therefore, it can be used in a combustible gas environment because it does not generate sparks electrically. It can contact a human safely because electric leakage is not possible [1, 2]. If the device is fabricated from non-magnetic material such as plastics, it can potentially be utilized in an MRI environment [3, 4]. Moreover, if the device incorporates many elastic inflatable chambers, it can generate complicated motions and have high passive adaptability [5, 6]. Such robots are called soft robots. A soft robot with high passive adaptability allows contacting complicatedshaped objects or fragile objects without the need for complicated control [7]. Of course, there are other operating principles of soft robots, such as chemical reactions

*Correspondence: takayama.t.aa@m.titech.ac.jp

Tokyo Institute of Technology, 2-12-1(13-13) Ookayama, Meguro-ku, Tokyo 152-8552, Japan or static electricity, but pneumatic actuation is realistic for practical use for now [8].

The operating principle of many pneumatic-driven robots uses periodic inflation and deflation of the air chambers inside them. If the motion is simple, such as the opening and closing motion of a robot hand, it can be driven by an air chamber supplied by a single air pressure source [9]. However, to generate continuous motion, such as the locomotion of a mobile robot or the rotation of a pneumatic-driven motor, some chambers that can be inflated and deflated periodically are required $[3,4,10-14]$. Therefore, these air chambers in such devices are equipped with individual air tubes that supply air pressure to the air chambers. Usually, the air pressures supplied to the air tubes are independently controlled by solenoid valves. For some soft robots, such solenoid valves need to be placed far from the workspace. For example, an in-pipe locomotive device cannot be equipped with solenoid valves because the solenoid valves are too large for the pipe. Further, a solenoid valve can generate electric sparks. Thus, it cannot be used near a gas pipe. Moreover, the pneumatic-driven motor 
developed for use under an MRI environment cannot utilize an electromagnetic device near the workspace. In such cases, long air tubes are required to drive the robot. This causes a large delay in the inflation and deflation of the air chambers because of the compressibility of air. As a result, the soft robot cannot generate quick motion. In such cases, a self-excited mechanism that can be driven by continuous airflow is effective. Some selfexcited pneumatic-driven devices have been developed. Pneumatic-driven motors that generate continuous rotational motion and can be used just like an electric motor have been developed $[15,16]$. A simple mechanism that uses the buckling motion of a bent tube to generate a self-excited vibration motion has been developed [17]. To supply air pressure to two different air chambers periodically, a device that uses a periodic jump of a magnet has been developed [18].

We are proposing a soft robot called a bundled tube locomotive device [19-21]. It is composed of several inflatable tubes that are bundled and periodically inflated and deflated. The tubes are bundled into helically twisted, 3-braided, or 6-braided shapes, and the device generates a helical rolling motion or winding motion based on its bundled shape and the sequence in which the tubes are inflated to move inside a narrow pipe. Its body has high elasticity and adapts against the inside pipe wall passively. Therefore, it can move inside pipes of different diameters and can pass through an elbow part without requiring complicated control. Moreover, the twisted-type bundled tube locomotive device can be fabricated by an extrusion molding machine continuously. Therefore, it is suitable for mass production, which can lower the device cost, turning it into a disposable device.
On the other hand, if the device becomes long, as shown in Fig. 1a, its inflatable air chambers also become long, and the amount of air to be supplied increases, too. Thus, both the frequency of the periodic inflation and deflation motion and its progress velocity decrease. In such a situation, connecting a short bundled tube locomotive device to non-inflatable air tubes that supply air pressure to the device is effective, as shown in Fig. 1b. However, if a pipe to be explored becomes long, the air-supply tube becomes long, too. This causes a large delay in the arrival of the pressure to the front tip of the device and also slows down the frequency of the periodic motion. To overcome this problem, a device that can be dragged inside the pipe and driven by continuous airflow to switch its output port periodically by selfexcited motion, as shown in Fig. 1c, is effective. However, the existing self-excited devices $[17,18]$ cannot be used for devices that need to change more than three output ports. Therefore, in this paper, we have proposed a compact self-excited airflow passage changing device that can be dragged into a pipe to drive our bundled tube locomotive device.

The rest of this paper is organized as follows. In "Method of self-exciting" section, we explain the mechanism and self-excitation principle of the device. In "Experimental results" section, we describe the experimental confirmation of the characteristics of the device. In "Discussion to adjust the output pressure and selfexcitationfrequency" section, based on the experimental results, we discuss the method to adjust the output pressure and self-excitation frequency, and the actual soft robot is connected to the developed device to confirm that the device can operate an in-pipe mobile soft robot.
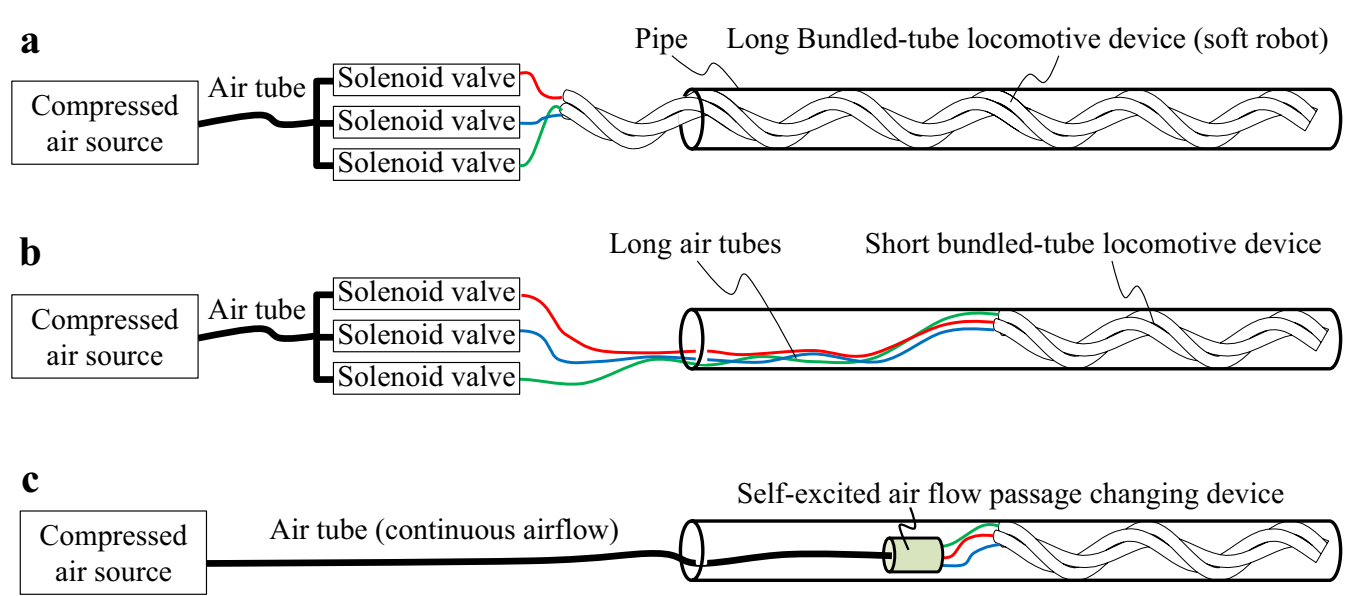

Fig. 1 Problem associated with a long traveling soft robot. a Problem associated with long soft robot. b Problem when air supply tubes become long. c Concept of the self-exciting airflow passage changing device 


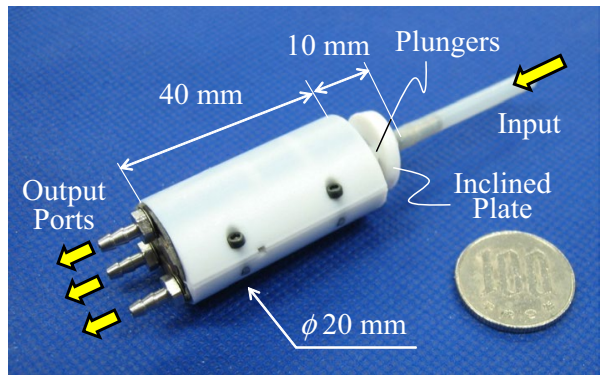

Fig. 2 Overview of the developed device

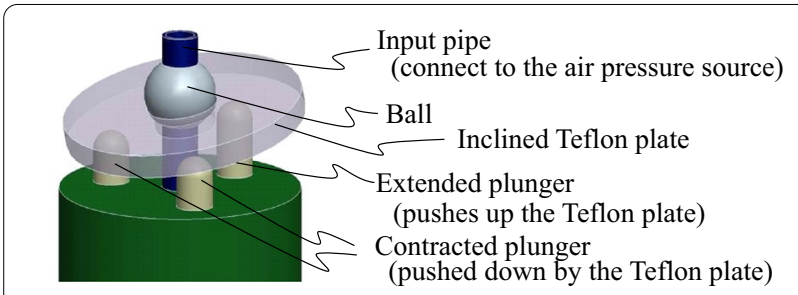

Fig. 3 Inclined plate to restrict the motion of the plungers

In "Conclusion" section, we provide concluding statements and outline future plans.

\section{Method of self-exciting}

The developed self-excited flow passage changing device is shown in Fig. 2. It has an input port and three output ports on opposite sides of the cylindrical body. By applying air pressure to the input port, the device periodically changes the airflow passage inside it to switch between the output ports. The bundled tube locomotive device is connected to the output ports, and its air chambers are inflated and deflated periodically. Three concentric cylinders are arranged inside the cylindrical body, which also houses three plungers. The heads of the plungers project to the input port side as shown in Fig. 3 and they are pushed against an inclined circular plate. The plate is made of Teflon to minimize friction and has a bowlshaped cavity. The cavity fits to a ball attached to the pipe of the input port so that the plate can rotate freely around the center of the ball. Therefore, when one plunger extends and pushes the plate up, the plate pushes down two other plungers, contracting them. Fig. 4 shows the cross section of an elbow part of a $25 \mathrm{~A}$ pipe and the size of the device [22]. The device is designed to pass through the elbow part, and the diameter and length of the main cylindrical part are 20 and $40 \mathrm{~mm}$, respectively.

Three concentric cylinders are arranged inside the device. The schematic diagram of a cross section of a plunger is shown in Fig. 5, where (a) and (b) show the

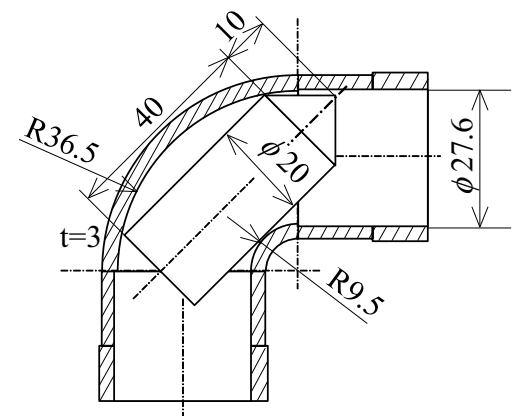

Fig. 4 Cross section of an elbow part of 25A pipe
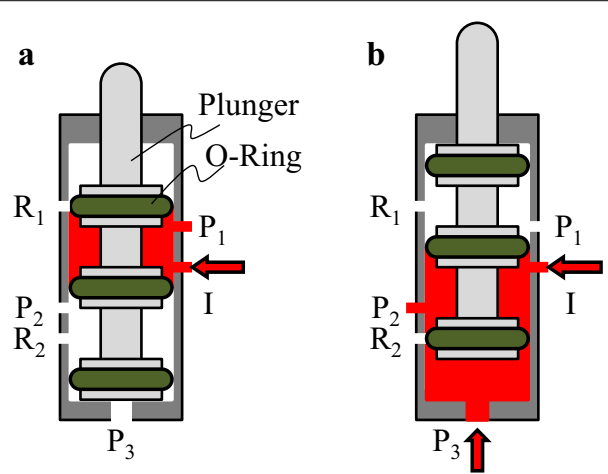

Fig. 5 Schematic diagrams of a plunger. a The plunger is contracted. b The plunger is extended

plunger in the contracted and extended, respectively. The cylinder has an input port that is connected to an air pressure source, two relief ports $R_{1}$ and $R_{2}$ that are released to atmospheric pressure, and three connecting ports $\mathrm{P}_{1}, \mathrm{P}_{2}$, and $\mathrm{P}_{3}$ that are connected to the other cylinders. When the plunger is contracted as shown in Fig. 5a, the plunger is not moved by the air pressure, and the pressure at port $\mathrm{P}_{1}$ becomes high. When air pressure is applied to port $P_{3}$, the plunger is pushed up as shown in Fig. $5 \mathrm{~b}$, and the pressure at port $\mathrm{P}_{2}$ becomes high.

Fig. 6 shows the working principle of the device. The first suffix indicates the number of the cylinder, and the second suffix denotes the number in the port name, as shown in Fig. 5. In reality, three concentric cylinders, $\mathrm{C}_{1}, \mathrm{C}_{2}$, and $\mathrm{C}_{3}$, are arranged, but in this figure, they are drawn schematically. Therefore, the flow channels of the right end $\alpha$ and $\beta$ are connected to the flow channels of the left end $\alpha$ and $\beta$. From now on, $n$ denotes the cylinder number, and $n+1$ and $n-1$ denote the neighboring cylinder numbers. However, in the case of $n=3, n+1$ denotes cylinder number 1 , and in the case of $n=1$, $n-1$ denotes cylinder number $3 . \mathrm{P}_{n 1}$ and $\mathrm{P}_{(n-1) 3}$ are connected by flow channel $\mathrm{F}_{n(n-1)} . \mathrm{P}_{n 2}$ and output port $\mathrm{O}_{(n+1)}$ are connected by flow channel $\mathrm{F}_{n(n+1)}$. Between port $\mathrm{P}_{n 3}$ and output port $\mathrm{O}_{n}$, there is a throttle valve $\mathrm{V}_{n}$ 

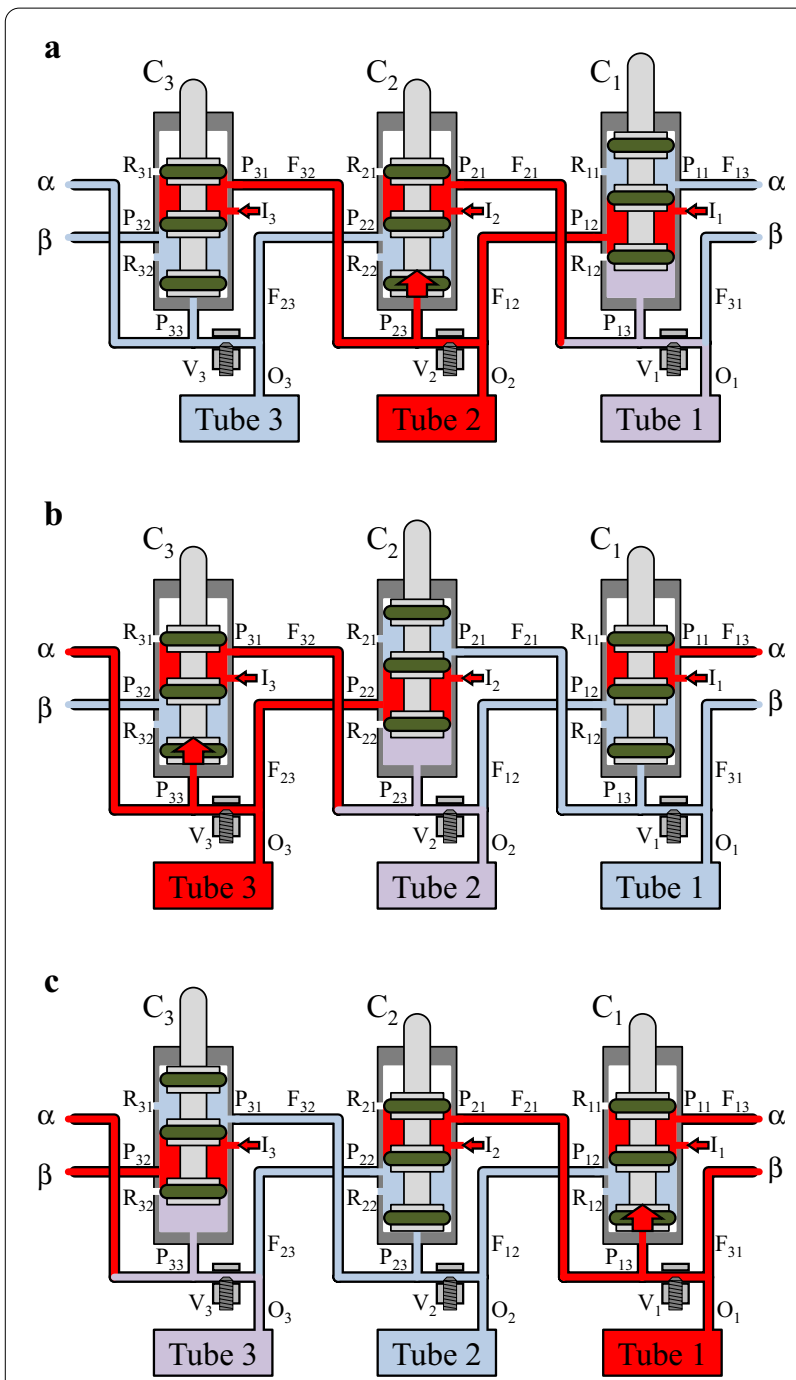

Fig. 6 Principle of self-excited motion generation. a-c show the states with plungers in $C_{1}, C_{2}$, and $C_{3}$ extended, respectively

that can narrow the flow channel by tightening a screw. At the output ports, the air chambers of the soft robot are connected.

When the plunger of $\mathrm{C}_{1}$ is pushed up, plungers of the other cylinders are pushed down by the inclined plate, as shown in Fig. 6a. The input air pressure from $\mathrm{I}_{1}$ and $I_{3}$ pressurizes tube 2 via $P_{12}$ and $P_{31}$, respectively. When tube 2 is inflated sufficiently and the pressure inside it becomes large, the pressure applied to port $\mathrm{P}_{23}$ pushes up the plunger in $C_{2}$. In this condition, the applied pressure to $I_{2}$ is connected to $R_{32}$ via $P_{21}$ and $P_{32}$. Therefore, the pressure at $\mathrm{P}_{13}$ becomes low. As a result, the plunger of $\mathrm{C}_{2}$ is pushed up and the plunger of $\mathrm{C}_{1}$ is pushed down by the inclined plate to reach the state shown in Fig. $6 \mathrm{~b}$. Then, $\mathrm{O}_{2}$ is connected to $R_{12}$ via $P_{12}$, and thus tube 2 is

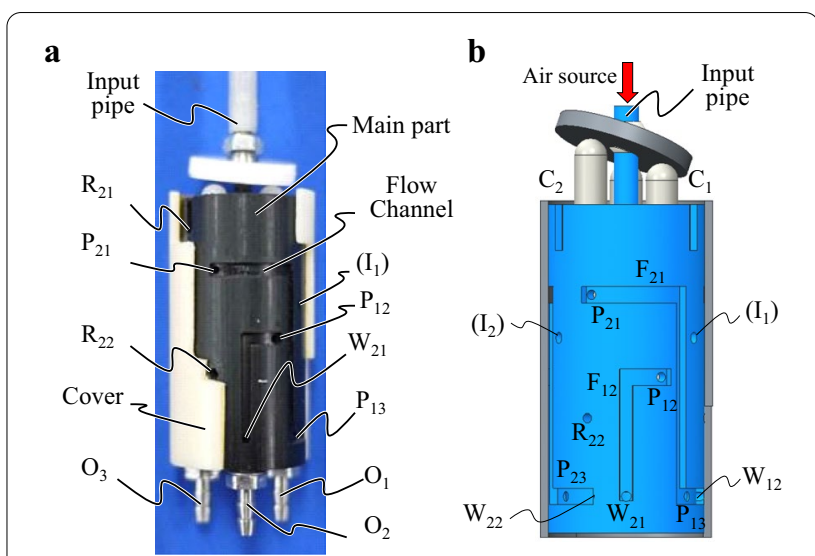

Fig. 7 Actual design of the cylindrical main part and its flow channels. a Photo of the part. $\mathbf{b}$ CAD graphic to show the ports and the flow channels

deflated. Similarly, when tube 3 is inflated sufficiently, the device changes its state, as shown in Fig. 6c. By changing its states in this way, the device can generate continuous self-oscillatory motion and change its airflow passage to inflate the three tubes periodically.

Throttle valve $V_{n}$ is introduced to control the selfexcitation frequency. For example, when the state changes from Fig. $6 a, b$, the plunger of $C_{1}$ needs to be pushed down. The air pressure in the cylinder needs to be released to the atmosphere from $R_{32}$ via $P_{32}$. On the other hand, the air pressure is still supplied from $\mathrm{I}_{2}$ via $\mathrm{P}_{21}$. Therefore, if the flow line is made narrow by $\mathrm{V}_{1}$, the effect of the supplied air pressure from $\mathrm{I}_{2}$ becomes dominant, and it resists the pushing down of the plunger. As a result, the frequency of the self-excited motion may decrease.

The actual design is shown in Fig. 7. Grooves are dug on the side surface of the cylindrical main part, and they are covered to operate as flow channels. Inside the main part, three holes are drilled from the bottom surface to form the cylinders, and the flow channels and the cylinders are connected by holes drilled from the side surface. At the central axis of the main part, a pipe is inserted and an air tube is connected to supply air pressure. The cross sections of Fig. 7 are shown in Fig. 8. A hole $\left(\mathrm{I}_{n}\right)$ is drilled from the side surface that reaches the central axis of the main part so that it connects the input pipe and the cylinders to become input port $\mathrm{I}_{n}$, as shown in Fig 8a. Hole $\left(\mathrm{I}_{n}\right)$ is closed by the cover so that it does not work. Fig. 8b shows the cross section at relief port $\mathrm{R}_{n 1}$. The flow channels connected to $R_{n 1}$ are connected to the top surface of the cylindrical main part to evacuate the air. Fig. 8c shows the cross section at input port $\mathrm{I}_{n}$. It is seen that the input ports are drilled from side surface up to the central axis of the main body. Fig. $8 \mathrm{~d}$ shows the cross section at 

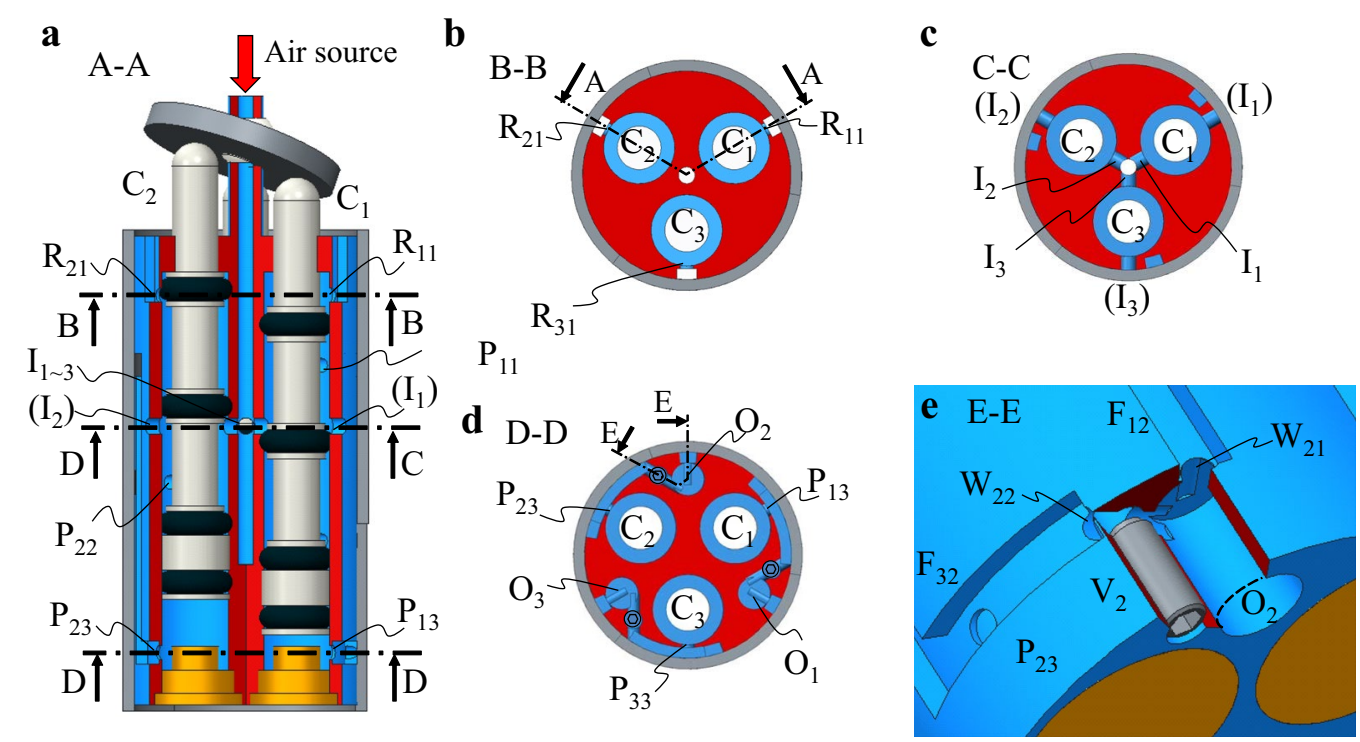

Fig. 8 Cross-sections of the main part. a Cylinders and plungers. b Cross section at the relief ports. $\mathbf{c}$ Cross section at the input ports. $\mathbf{d}$ Cross section to show the connection of the flow channels at output ports. e Cutaway image of output port to show the fitted throttle valve

port $\mathrm{P}_{n 3}$. Output ports $\mathrm{O}_{n}$ are equipped with tube fittings to connect the soft robot. To help the reader understand the connection of flow channels $F_{32}$ and $F_{12}$, a cutaway image of output port $\mathrm{O}_{2}$ is also shown in Fig. 8e. Flow channel $\mathrm{F}_{12}$ and $\mathrm{O}_{2}$ are connected by hole $\mathrm{W}_{21}$, and flow channel $\mathrm{F}_{32}$ and $\mathrm{O}_{2}$ are connected by hole $\mathrm{W}_{22}$. To control the flow amount in hole $\mathrm{W}_{22}$, a set screw is fitted to work as throttle valve $\mathrm{V}_{2}$.

\section{Experimental results}

In this mechanism, until the air pressure in the air chamber of the soft robot connected to output port $\mathrm{O}_{n}$ becomes high enough, the air pressure of port $\mathrm{P}_{n 3}$ that pushes the plunger also remains low, and the plunger does not start to move. When sufficient air is supplied to the air chamber, the pressure that pushes the plunger becomes high, the plunger starts to move, and its airflow passages change. Therefore, the device's frequency of selfexcitation may be affected by the volume of the air chamber of the soft robot. To apply the proposed device to many types of soft robots, it should be possible to change the output pressure and the self-excitation frequency arbitrarily without modifying the design considerably.

\section{Effect of the volume of the air chambers}

First, we confirmed the effect of the volume of the air chambers. In the experiment, we compared its behaviors when non-deformable air chambers of volumes ranging from 0 to $10,000 \mathrm{~mm}^{3}$ are attached to the output ports. The air pressure applied to the input port is $0.4 \mathrm{MPa}$.
Air pressure gauges are attached to the chambers to measure the pressure of the output port. The results are shown in Fig. 9, where (a), (b), and (c) show air chambers of volumes $0 \mathrm{~mm}^{3}$ and $10,000 \mathrm{~mm}^{3}$, and the relationship between the volume of the attached air chambers and the frequency of the self-excitation. The output pressures are almost constant at $0.3 \mathrm{MPa}$, and the frequency decreases when the volume of the air chamber increases, which is in line with our expectations.

\section{Effect of the flow amount of the supplied air}

We considered that by changing the flow amount supplied to the input port, the self-oscillation frequency will also be changed. We attached a throttle valve (KOGANEI TVU4 [23]) to the air-supplying tube and measured the frequencies and the output pressure when the throttle valve is rotated. The supplied air pressure is $0.4 \mathrm{MPa}$, and air chambers of volume $10,000 \mathrm{~mm}^{3}$ are connected to the output ports. The experimental results are shown in Fig. 10. As the throttle valve is rotated by 4.5 to 5.5 rounds, the frequency and output pressure increase correspondingly, and if the number of rotation rounds exceeds 5.5, saturation is reached. Moreover, if the number of rotation rounds is smaller than 4 , the self-oscillation motion becomes unstable. Therefore, the motion frequency and the output pressure cannot be independently controlled by varying the flow speed alone. Moreover, the controllable range of this technique is small. 
a Volume $0 \mathrm{~mm}^{3}$

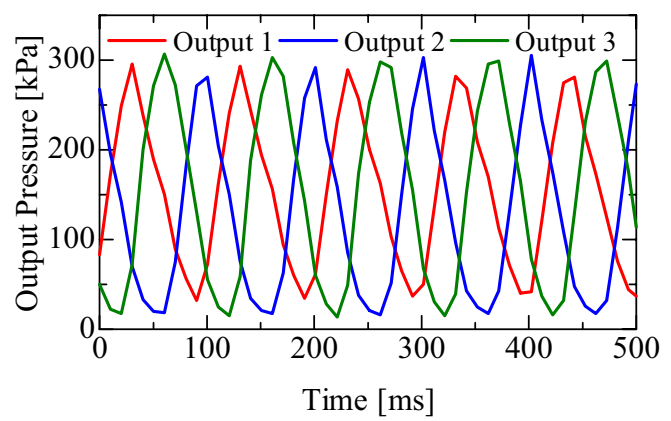

b Volume $10,000 \mathrm{~mm}^{3}$

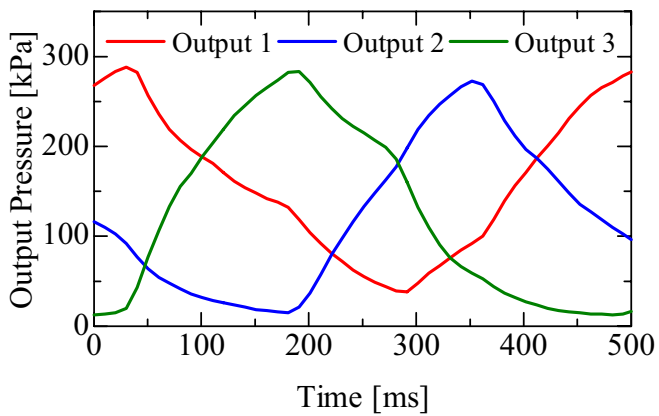

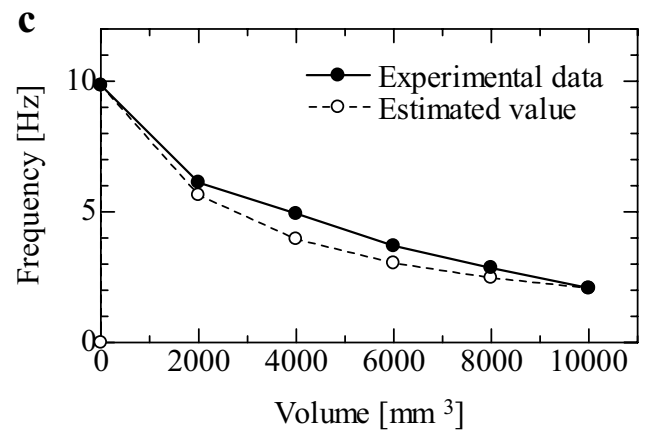

Fig. 9 Frequencies affected by the volume of the chambers. a Volume of the attached chamber is $0 \mathrm{~mm}^{3}$. $\mathbf{b}$ Volume of the attached chamber is $10,000 \mathrm{~mm}^{3}$. c Relationship between the volume of the attached air chambers and frequency of self-excitation
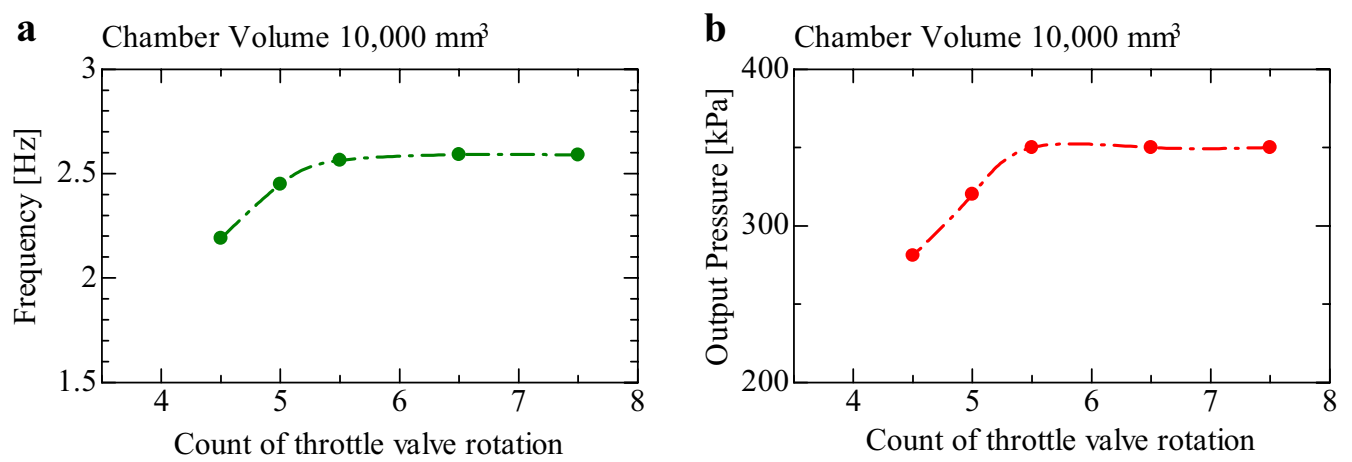

Fig. 10 Frequencies and output pressures affected by the amount of the input flow. a Relationship between the rotation of the throttle valve and the frequency. $\mathbf{b}$ Relationship between the rotation of the throttle valve and the output pressure

\section{Effect of the pressure of the supplied air}

Next, we varied the input pressure and measured the motion frequency and the output pressure. In this experiment, air chambers of volume $2000 \mathrm{~mm}^{3}$ are connected to the output ports so that the frequency does not become too slow, which would make the motion unstable. The experimental results are shown in Fig. 11. It is observed that increasing the applied input pressure produces a corresponding increase in the motion frequency and the output pressure. Moreover, if the input pressure becomes lower than $250 \mathrm{kPa}$, the motion becomes unstable. These results also show that we cannot control the frequency and the output pressure independently by controlling the input pressure alone.

\section{Effect of the fitted throttle valves}

Next, we measured the effect of the throttle valves fitted between port $\mathrm{P}_{n 3}$ and output port $\mathrm{O}_{n}$. We compared the status when the three valves were fully opened with that when the set screws were closed for 

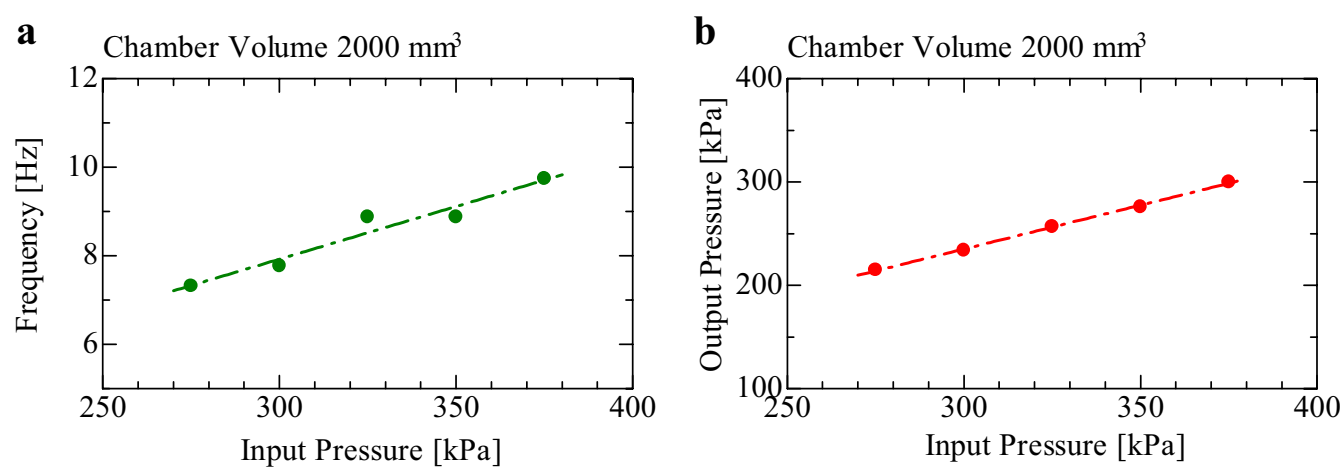

Fig. 11 Frequencies and output pressures affected by the input pressure. a Relationship between the input pressure and the frequency. $\mathbf{b}$ Relationship between the input pressure and the output pressure

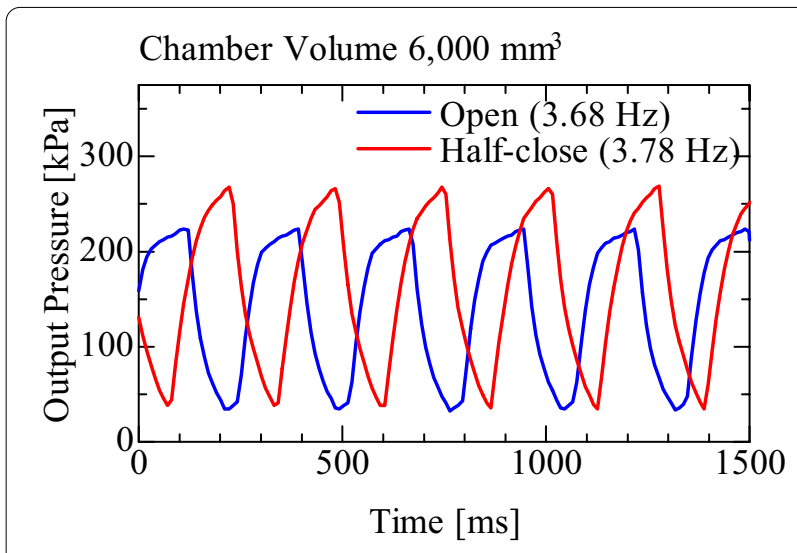

Fig. 12 Effect of the internal throttle valve

five rounds that make the valve about half-closed. In this experiment, air chambers of volume $6000 \mathrm{~mm}^{3}$ are connected to the output ports, and the input flow amount is adjusted so that the output pressure becomes $200 \mathrm{kPa}$. This setting is so close to the border that the motion becomes unstable, because we expected that the device could overcome this border by using the inner throttle valves. The experimental result is shown in Fig. 12. We considered that closing the valve may decrease the self-excitation frequency. However, the result shows that when the throttle valve is half-closed, the frequency is not much affected, but the output pressure is clearly increased.

\section{Discussion to adjust the output pressure and self-excitation frequency} Mechanism of the unstable motion

The developed device uses O-rings to seal the cylinder and plunger, which causes friction. The friction heat

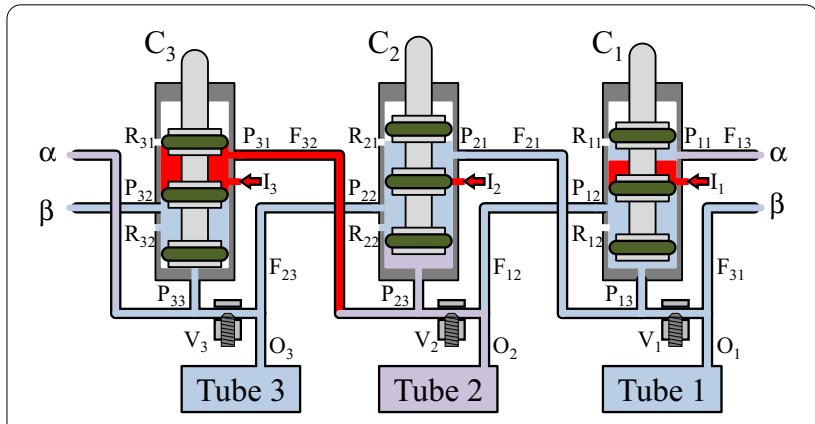

Fig. 13 Unstable states of the plungers

can be cooled by the air passing through the cylinder. We considered that this friction causes unstable motion when the motion frequency is reduced. If the supplied flow amount is small, the plunger generates stick-slip motion, and the plungers stop in the middle of changing state, as shown in Fig. 13. In this condition, the input pressure from $I_{1}$ is released to $R_{11}$, and $I_{2}$ is closed by the O-ring. An effective way to overcome this problem is to make the cylinder hole and plunger accurately so that it has a slide fit tolerance. A threshold mechanism that has a stable point at the upper or lower side of the plunger motion is also effective.

\section{The range of output pressure}

In all the experiments, the output pressures are smaller than the input pressure and slightly larger than the atmospheric pressure. The device changes the flow channels by the antagonistic drive of the two plungers. In Fig. 6a, the plungers in $C_{1}$ and $C_{2}$ are driven antagonistically. If the pressure of $\mathrm{P}_{23}$ overcomes that of $\mathrm{P}_{13}$, even if the pressure of $\mathrm{P}_{23}$ does not become equal to the input pressure, the plungers start to move, and its state changes 
to that shown in Fig. 6b. This is a mechanism of the difference between the input pressure and output pressure. Therefore, we need to supply higher pressure than the required pressure to drive a soft robot. Moreover, if the motion frequency becomes high, the minimum value of output pressure is increased. Because the flow channels are narrow, it becomes resistant to vent the air from the attached air chamber completely before its state changes. This problem occurs even if solenoid valves are used. Therefore, we compared the developed device with a usual solenoid valve. We used solenoid valves (SMC V100) that are smaller than a $25 \mathrm{~A}$ pipe. Fig. 14 shows the experimental result. If the deformation of the soft robot depends on the difference between the maximum and minimum pressures, the developed device will work better than the solenoid valve.

\section{Saturation of the flow amount of the air}

The experimental results in "Effect of the flow amount of the supplied air" section show that if the number of rotation rounds of the throttle valve exceeds 5.5, saturation is reached. In this condition, we measured the flow amount, which was about $40 \mathrm{~L} / \mathrm{min}(\mathrm{ANR})$. We considered that it reached a choked flow. The flow amount of a choked

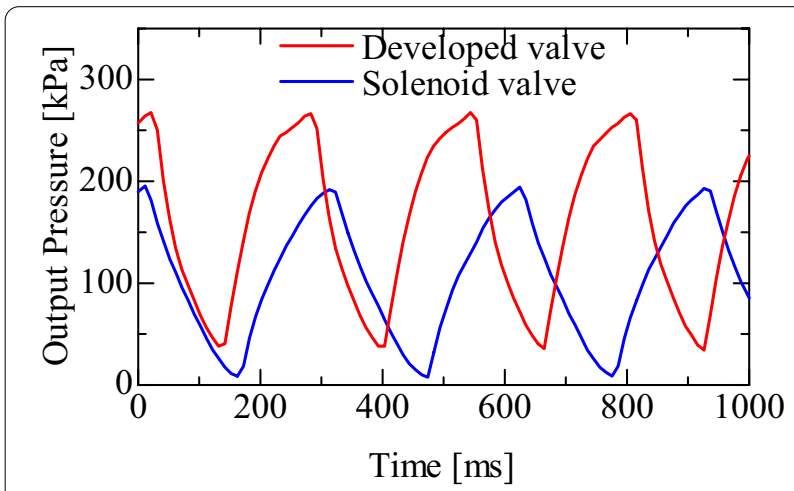

Fig. 14 Comparison of the developed device and the solenoid valve flow is obtained by $Q=120 S(P+0.1)$, where $Q, S$, and $P$ are the flow amount, effective sectional area, and pressure difference, respectively. From $Q=40 \mathrm{~L} / \mathrm{min}$ and $P=0.4 \mathrm{MPa}, S=0.67 \mathrm{~mm}^{3}$ is obtained. In the developed device, the most narrow part of the flow channels is the ports drilled from the side surface to the cylinders. The diameter of the holes is $1 \mathrm{~mm}$, and its area is $0.78 \mathrm{~mm}^{3}$. The actual flow channels have complicated shapes, and the airflow needs to pass through many small holes to arrive at the air chamber, but the values of these areas seem close. Therefore, we consider that the holes define the maximum flow amount of air in the developed device.

\section{Relation between the motion frequency and the flow amount}

The motion of the proposed device is strongly affected by the air chambers attached to the output port. Moreover, the deformation of the usual soft robot has nonlinear characteristics. Therefore, it is difficult to predict the motion of the device. On the other hand, if the attached chamber is non-inflatable, the motion frequency of the developed device depends on the flow amount of the supplied air. If the volume of the attached chamber is $0 \mathrm{~mm}^{3}$, the frequency is $9.84 \mathrm{~Hz}$, as shown in Fig. 9. Therefore, the time for changing the state is $t_{c}=1 / 9.84 / 3=0.0339 \mathrm{~s}$. To simplify the model, we assume that the flow amount to the air chamber is proportional to the difference between the pressures of the input port and the air chamber, as shown in Fig. 15a. Therefore $\quad Q_{\text {in } e}=Q_{\text {in } s} \times\left(P_{\text {in }}-P_{e}\right) /\left(P_{\text {in }}-P_{s}\right)$, where $Q_{\text {in } s}, Q_{\text {in } e}, P_{i n}, P_{s}$, and $P_{e}$ are the flow amount of start to flow in, the flow amount of finish to flow in, the input pressure, the output pressure of start to flow in, and the output pressure of finish to flow in, respectively. The time period for which air needs to be supplied to a chamber can be calculated by $\left(Q_{\text {in } \_}+Q_{\text {in_e }}\right) t_{i} / 2=V\left(P_{e}-P_{s}\right) / 0.1$, where $t_{i}$ and $V$ are the time of inflating and the volume of the chamber, respectively. Thus, the time for one periodic motion

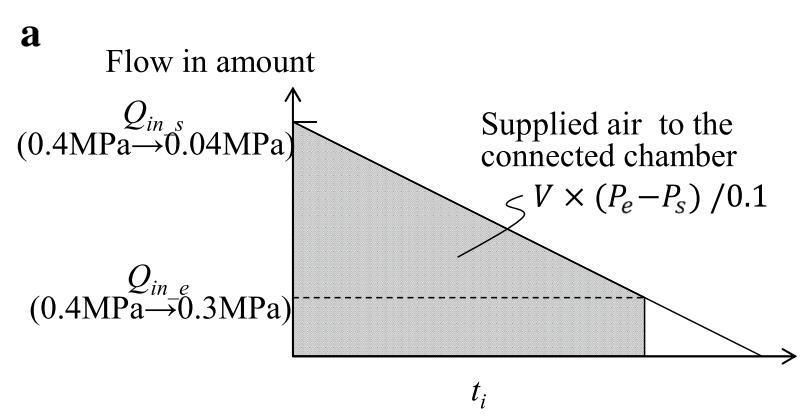

b

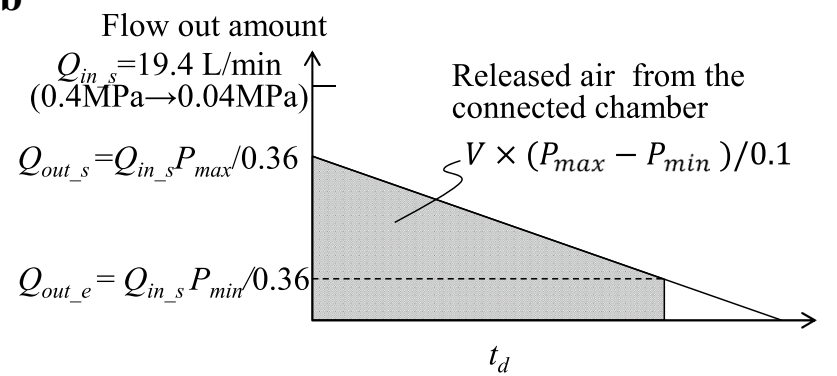

Fig. 15 Estimation method of the flow in/out air. a In case of flow in. $\mathbf{b}$ In case of flow out 
can be obtained by $T=\left(t_{c}+t_{i}\right) \times 3$. In the experiment shown in Fig. 9b, $P_{i n}, P_{s}, P_{e}, V$, and the motion frequency are $0.4 \mathrm{MPa}, 0.04 \mathrm{MPa}, 0.3 \mathrm{MPa}, 10,000 \mathrm{~mm}^{3}$, and 2.08 $\mathrm{Hz}$, respectively. From these equations and experimental data, we can obtain $t_{i}=1 / 2.08 / 3-t_{c}=0.126 \mathrm{~s}$ and $Q_{s}=323 \times 10^{3} \mathrm{~mm}^{3} / \mathrm{s}(\simeq 19 \mathrm{~L} / \mathrm{min})$. Therefore, we considere that the supplied air of $19 \mathrm{~L} / \mathrm{min}$ (ANR) contributes in pressurizing the chamber, and $21 \mathrm{~L} / \mathrm{min}(\mathrm{ANR})$ is released from the relief ports, as shown in Fig. 6. From this distribution of the flow amount, the theoretical frequency with the volume of the attached chambers can be estimated, as shown in Fig. 9, and it seems reasonable.

Similarly, we can obtain the relations of the maximum and minimum pressures of the output port, frequency, and volume of the air chamber, in case the output pressure is changed. We assume that the resistance of the flow channel between the input port and the output port is the same as that between the output port to the release port so that the proportion of the flow amount depending on the pressure becomes the same. The relation can be calculated by $\left(Q_{\text {out_s }}+Q_{\text {out_e }}\right) t_{d} / 2=V\left(P_{\max }-P_{\min }\right) / 0.1$, where $Q_{\text {out_s }}, Q_{\text {out_e }}, t_{d}, P_{\max }$, and $P_{\min }$ are the flow amount of start to flow out, the flow amount of finish to flow out, time of deflating, the maximum output pressure, and the minimum output pressure, respectively, as shown in Fig. 15b. To release air from the chamber, two of three states are used, as shown in Fig. 6, with one state supplying and releasing air simultaneously (Fig. 6a Tube 1). Therefore, we treat 1.5 states are used for releasing time to simplify the model. For example, in Fig. 12, the maximum pressure, frequency, and volume of the chamber are $0.22 \mathrm{MPa}, 3.68 \mathrm{~Hz}$, and 6,000 $\mathrm{mm}^{3}$, respectively. Thus, the time to release the air is $t_{d}=1 / 3.68 / 3 \times 1.5-t_{c}=0.102 \mathrm{~s}$. From these values, $P_{\text {min }}$ is calculated as $P_{\text {min }}=0.03 \mathrm{MPa}$, and it seems not different largely from the measured minimum output pressure $(0.034 \mathrm{MPa})$. The difference may be caused by the treatment of the state in that air is being supplied and released simultaneously. The maximum output pressure is hard to calculate because it depends on the antagonistic motion of two plungers. However, if the required maximum pressure and frequencies are decided, the minimum pressure can be roughly estimated.

\section{Method to independently adjust the output pressure and self-excitation frequency}

The experimental results show that the self-excitation frequency and the output pressure can be adjusted independently in the following way. First, set the input pressure so that it is slightly larger than the pressure required to deform the soft robot. Next, by controlling the amount of the input flow, adjust the frequency. Next, by tightening the inner throttle valve, increase the output pressure until it reaches the pressure required to deform the soft robot. If the output pressure cannot reach the required pressure, the input pressure is increased, and the adjusting procedure is repeated. However, the experimental results show that this control method does not have a large controllable range. Therefore, we propose another possible control method, which is described in Appendix.

\section{Confirm the proposed adjustment method by applying the developed device to a soft robot}

We conducted experiment to confirm that the developed device can drive an actual soft robot. For the soft robot, we used our developped twisted-type bundled tube locomotive device (Fig. 16a). The diameter of the device is 6 $\mathrm{mm}$ and the cross-sectional design is shown in Fig. 16b. The device is fabricated by extrusion processing. The developed molding die is shown in Fig. 16c. Heated polyvinyl chloride (PVC) is extruded from the slit of the die and twisted before the PVC is cooled for curing to form the twisted air chambers. The developed bundled tube locomotive device requires pressure of $250-350 \mathrm{kPa}$ to
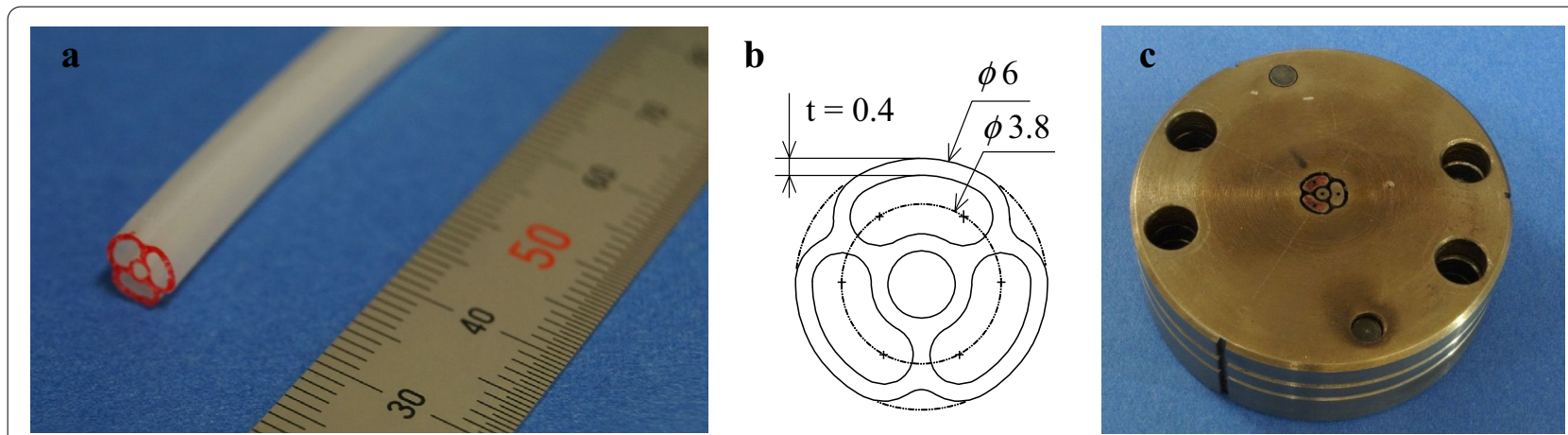

Fig. 16 Used soft robot. a Cut surface of the actual device. $\mathbf{b}$ Design of the mold. $\mathbf{c}$ Developed mold for the extrusion process to make the soft robot 


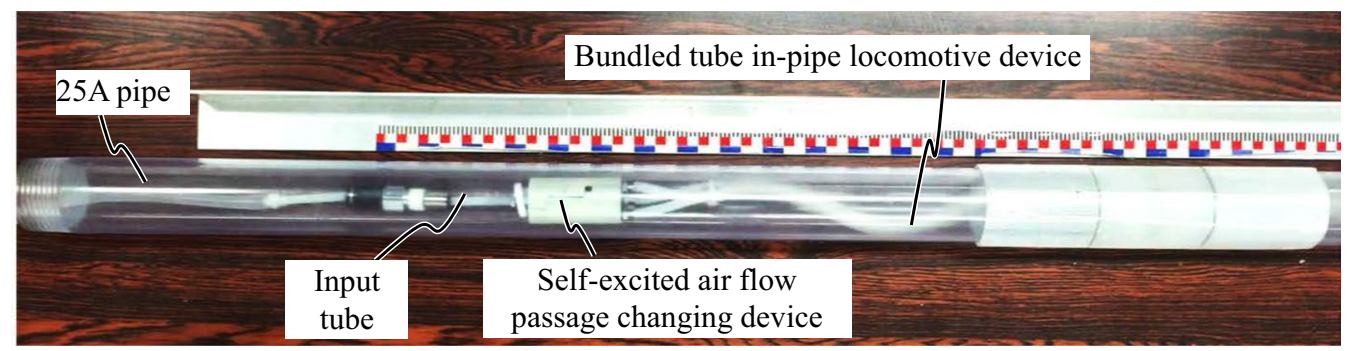

Fig. 17 Experiment to drive the device in a 25A pipe

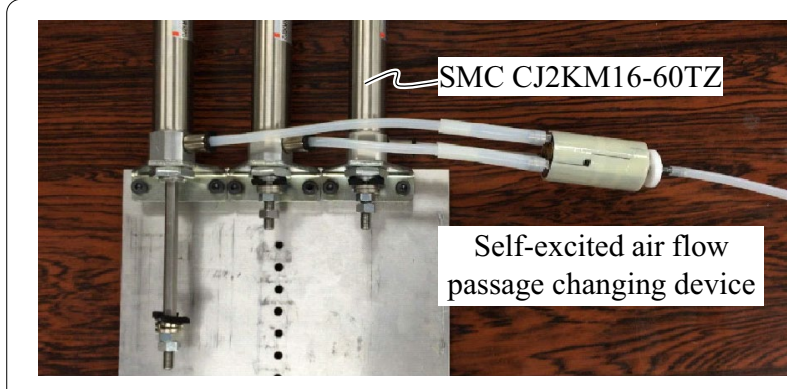

Fig. 18 Experiment to actuate air cylinders

produce enough deformation to move in a $25 \mathrm{~A}$ pipe. We connected the bundled tube locomotive device of approximately $30 \mathrm{~cm}$ to the self-excited air flow passage changing device, and adjusted the inner throttle valves and the input pressure so that the device can progress in the $25 \mathrm{~A}$ pipe by trial and error, based on the adjustment method proposed in the upper section. The device generated a self-excited motion of approximately $4 \mathrm{~Hz}$, and it could move at approximately $20 \mathrm{~mm} / \mathrm{s}$ by dragging the airflow passage changing device (Fig. 17). On the other hand, it could not pass through the elbow of the 25A pipe, because the tube fittings of the input and output ports of the airflow passage changing device are long, and they got caught at the elbow.

\section{Confirm the general purpose use}

Moreover, to confirm the general-purpose applications of this device, we experimented by connecting air cylinders (SMC CJ2KM16-60TZ) to the output ports (Fig. 18). The air cylinder has a spring inside it to extend the rod, and it contracts when air pressure is applied. We managed to drive it at $1.3 \mathrm{~Hz}$ without causing any unstable motion. This result is slower than the result shown in Fig. 10. We consider that this is caused by the spring in the air cylinder. In the developed device, two plungers push each other antagonistically. If noninflatable air chambers are connected to the output ports, the pressure of the output port decreases with time as shown in Fig. 19. In this case,

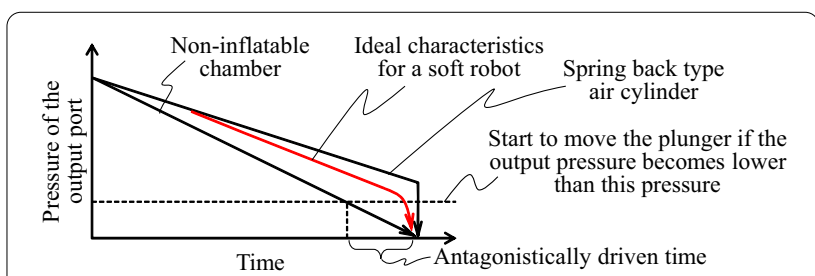

Fig. 19 Change of the pressure at the output port with time when the air is released

the plungers start to move before the output pressure decreases to 0 . Therefore, the use of two plungers maintain an antagonistic movement until the output pressure decreases to 0 . This mechanism causes unstable motion. On the other hand, when air is released from the springback type air cylinder, the chamber can maintain the pressure until it releases the air perfectly, and the pressure reduces to 0 in a moment. It shortens the duration of the antagonistic drive of two plungers. We consider this to be reason why it can work with such slow frequency. Further, we consider that if we can give a similar characteristic to a soft robot, as shown in Fig. 19, it can be actuated more stably.

\section{Conclusion}

The developed self-excited air flow passage changing device succeeded in generating self-excitation motion in lie with our expectations. On the other hand, contrary to our expectations, the inner throttle valve did not reduce the frequency, but it increased the output pressure. As a result, we could adjust the output pressure and the self-excitation frequency independently. The developed self-excited air flow passage changing device could drive our soft robot called the bundled tube in-pipe locomotive device, and the robot could make progress in a $25 \mathrm{~A}$ pipe by dragging the developed device itself into the pipe. However, it could not pass through the elbow part, because its tube fittings are 
large. In the future, we need to design smaller device including tube fittings so that the device can pass through the elbow part. Moreover, we will introduce additional inner throttle valves at the relief ports so that the device can adjust the output pressure and selfexcitation frequency over a larger range and attempt to apply this device to other soft robots for wide use. The deformation of the soft robots is usually nonlinear, and its characteristics are not uniform. Therefore, sometimes it needs to adjust the driving pressures of the chambers independently. By combining the inner throttle valves at the output ports and the additional throttle valves of the relief ports, it has a possibility to adjust the output pressure and motion timing of each plunger independently. The device was developed to drive our in-pipe robot, which requires a high pressure compared to the driving pressure for usual soft robots, and it needs only a one-directional progress motion. Therefore, our future work will focus on developing a device that can be driven by lower pressure is capable of generating a bidirectional motion.

\section{Appendix}

We expected the fitted throttle valves to reduce the selfexcitation frequency. However, it could not reduce the frequency. This is because not only does $V_{1}$ resistant to vent the pressure of cylinder $C_{1}$ to port $P_{32}$, but also $V_{2}$ becomes resistant to the flow from $I_{1}$ to $P_{23}$, which contributes to the increase in pressure of cylinder $C_{2}$, at the state shown in Fig. 6a. As a result, the balance of the antagonistic drive of the plungers in $C_{1}$ and $C_{2}$ is not changed significantly. To control the self-excitation frequency, we also propose another simple method of halfclosing the relief ports. If the cross-sectional area of relief port $\mathrm{R}_{n 2}$ becomes small, it needs a long time to relieve the air that pushes the plunger when the plunger is pushed down, and the frequency is expected to decrease. For the experiment, half the area of relief ports $R_{n 2}$ were covered by tape as shown in Fig. 20a, and the device was assembled. We applied an input pressure of $375 \mathrm{kPa}$ and adjusted the inner throttle valve so that the output pressure becomes $200 \mathrm{kPa}$. The experimental results are shown in Fig. 20b. The output pressures are the same, and the frequency significantly decreases when the relief ports are half-closed. Therefore, if a throttle valve is fitted at the relief port, it can control the self-excitation frequency and the output pressure independently over a larger range.

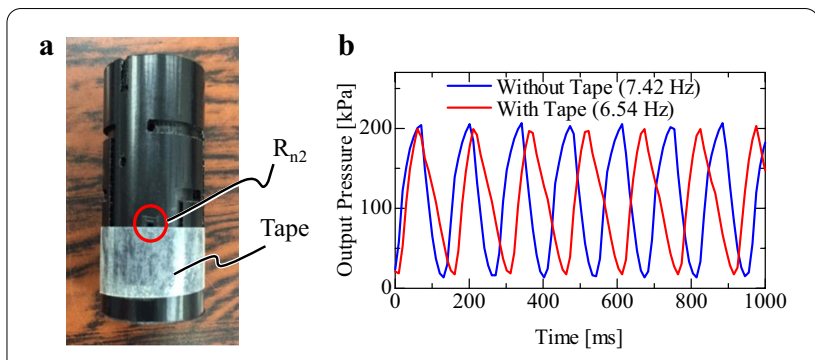

Fig. 20 Additional method to control the frequency. a Relief ports half closed by a tape. $\mathbf{b}$ Experimental results

\section{Authors' Contributions}

TT contributes to propose the basic idea and design of the experimental systems. YS contributes to develop the actual device and conduct the experiments.

\section{Funding}

This work was supported by JSPS KAKENHI under Grant 17 K06253.

Availability of data and materials

The datasets used and/or analyzed during the current study are available from the corresponding author on reasonable request.

\section{Declarations}

Competing interest

The authors declare that they have no competing interests.

Received: 28 May 2021 Accepted: 17 August 2021

Published online: 26 August 2021

\section{References}

1. Nomura K, Sato M, Takeuchi H, Minoru K, Ryoichi T, Ishii H, Takanishi A (2017) Development of in-pipe robot with extension hose and balloons. In: Proceedings of the 2017 IEEE international conference on mechatronics and automation (ICMA), pp 1481-1486

2. Yuichi N, Kazuki K, Shigeki T, Yuuta K, Takuya N (2014) Study of active catheter -development of the mechanism corresponding to a coronary arteries. In: Proceedings of the 2014 JSME conference on robotics and mechatronics, pp 3-205

3. Hiroyuki S, Hiroki K, Kenta K, Takeyoshi D, Ken M (2012) Mr-safe pneumatic rotation stepping actuator. J Robot Mechatron 24(5):820-827

4. Kazuo U, Isao E, Koichi S (2007) Development of nutation motors (4th report, development of small-sized and high torque pneumatic nutation motor by the ofw type bevel gears and principle of lever). Trans Jpn Soc Mech Eng (C) 73(730):1731-1736

5. Koichi S, Shoichi I, Hirohisa T (1992) Applying a flexible microactuator to robotic mechanisms. IEEE Control Syst 12(1):21-27

6. Shinichi H, Tomohiro M, Sadao K (2002) Prototyping pneumatic group actuators composed of multiple single-motion elastic tubes. J Robot Soc Jpn 20(3):299-306

7. Takanobu H, Watanabe Y, Ishihara H, Aizawa T, Ohura M (2005) Silkworm handling robot system. In: Proceedings of the 2005 IEEE international conference on robotics and automation, pp 2988-2993

8. Hidenori O, Yoshihito O (2014) Classification and research trend of soft actuators. J Jpn Soc Precis Eng 80(8):709-712

9. Wang Z, Torigoe Y, Hirai S (2017) A prestressed soft gripper: design, modeling, fabrication, and tests for food handling. IEEE Robot Autom Lett 2(4):1909-1916 
10. Ozaki K, Wakimoto S, Suzumori K, Yamamoto Y (2011) Novel design of rubber tube actuator improving mountability and drivability for assisting colonosocope insertion. In: Proceedings of the 2011 IEEE international conference on robotics and automation, pp 3263-3268

11. Shepherd RF, llievski F, Choi W, Morin SA, Stokes AA, Mazzeo AD, Chen X, Wang M, Whitesides GM (2011) Multigait soft robot. Proc Natl Acad Sci 108(51):20400-20403

12. Suzumori K (1993) Micro-walking robot driven by flexible microactuator. J Robot Mechatr0n 15(6):57-541

13. Toshiro N, Mitsuhiko K (2000) Development of in-pipe mobile robot using pneumatic soft-actuator. J Robot Soc Jpn 18(6):831-838

14. Jinwan L, Hyunjun P, Sunmoo M, Byungkyu K (2007) Pneumatic robot based on inchworm motion for small diameter pipe inspection. In: Proceedings of the 2007 IEEE international conference on robotics and biomimetics, pp 330-3335

15. Hitoshi K, Shigeo H (2003) Development of crown motor. Trans Jpn Soc Mech Eng (C) 69(677):117-125

16. Hideyuki T, Seiichi N, Ato K (2000) Versatile water hydraulic motor driven by tap water. In: Proceedings of the 2000 IEEE/RSJ international conference on intelligent robots and systems, p 2
17. Hideyuki T (2011) $\lambda$-drive: fluid powered drive by using buckling on a flexible tube and its application. J Robot Soc Jpn 29(6):488-491

18. Yuji M, Hideyuki T (2017) Soft compact valve inducing self-excited vibration and its application for a mobile robot. In: Proceedings of the 2017 JSME conference on robotics and mechatronics, pp 2-103

19. Takayama T, Takeshima H, Hori T, Omata T (2015) A twisted bundled tube locomotive device proposed for in-pipe mobile robot. IEEE/ASME Trans Mechatron 20(6):2915-2923

20. Takeshima H, Takayama T (2017) Geometric estimation of the deformation and the design method for developing helical bundled-tube locomotive devices. IEEE/ASME Trans Mechatron 23(1):223-232

21. Takeshima H, Takayama T (2018) Development of a steerable in-pipe locomotive device with six braided tubes. ROBOMECH J 5(1):1-11

22. Pocket Book of Japanese Industrial Standard. https://jis.jts-tokyo.com/ table-screwed-type-malleable-cast-iron-pipe-fittings-90deg-elbow-fitti ngs-standards/. Accessed 1 Apr 2021

23. Online catalog of KOGANEI. https://official.koganei.co.jp/product/THROT TLE_VALVES_ALL. Accessed 1 Jul 2021

\section{Submit your manuscript to a SpringerOpen ${ }^{\circ}$ journal and benefit from:}

- Convenient online submission

- Rigorous peer review

- Open access: articles freely available online

- High visibility within the field

- Retaining the copyright to your article

Submit your next manuscript at $\boldsymbol{\nabla}$ springeropen.com 